\title{
SPACES OF VECTOR FUNCTIONS THAT ARE INTEGRABLE WITH RESPECT TO VECTOR MEASURES
}

\author{
JOSÉ RODRÍGUEZ
}

(Received 18 January 2005; revised 27 July 2005)

Communicated by A. J. Pryde

\begin{abstract}
We study the normed spaces of (equivalence classes of) Banach space-valued functions that are Dobrakov, $S^{*}$ or McShane integrable with respect to a Banach space-valued measure, where the norm is the natural one given by the total semivariation of the indefinite integral. We show that simple functions are dense in these spaces. As a consequence we characterize when the corresponding indefinite integrals have norm relatively compact range. On the other hand, we also determine when these spaces are ultrabornological. Our results apply to conclude, for instance, that the spaces of Birkhoff (respectively McShane) integrable functions defined on a complete (respectively quasi-Radon) probability space, endowed with the Pettis norm, are ultrabornological.
\end{abstract}

2000 Mathematics subject classification: primary 28B05, 46G10; secondary 46A08, 46E40.

Keywords and phrases: Dobrakov integral; $S^{*}$-integral; McShane integral; Birkhoff integral; ultrabornological space; barrelled space.

\section{Introduction}

In the framework of integration of Banach space-valued functions defined on probability spaces, the notions of Birkhoff and (generalized) McShane integrability, that lie strictly between Bochner and Pettis integrability, have been widely studied in the last years, see $[3,24,11,9]$ and [25] among others. In a recent paper [26] we discussed the natural extensions of the Birkhoff and McShane integrals to the case of integration with respect to vector measures, comparing them with the Dobrakov (Bartle) integral $[1,6]$. The corresponding generalization of the Birkhoff integral was first considered by Dobrakov [8] under the name $S^{*}$-integral. Our purpose here is to analyze some

Partially supported by the grant BFM2002-01719 of MCYT (Spain) and a FPU grant of MECD (Spain). (C) 2007 Australian Mathematical Society 1446-7887/07 $\$$ A2.00+0.00 
aspects of the spaces of (equivalence classes of) vector-valued functions that are Dobrakov, $S^{*}$ or McShane integrable with respect to a vector measure, equipped with the norm given by the total semivariation of the indefinite integral. We refer the reader to Section 2 for the definitions.

The study of the spaces of (equivalence classes of) vector-valued integrable functions defined on a probability space, endowed with the norm given by the total semivariation of the associated indefinite integral (usually called the Pettis norm), was initiated by Birkhoff [2] and Pettis [23] in the thirties. Theorem 18 in [2] states that simple functions are dense in the space of Birkhoff integrable functions, but the analogous question for the Pettis integral remained open until Fremlin and Talagrand gave, in [13], a negative answer by showing an example of an indefinite Pettis integral without norm relatively compact range. Recall that a Pettis integrable function is the limit, for the Pettis norm, of a sequence of simple functions if and only if the range of its indefinite integral is norm relatively compact, see [19, Theorem 9.1]. On the other hand, it is also known that the indefinite Pettis integral of a McShane integrable function defined on a quasi-Radon probability space always has norm relatively compact range (Fremlin, see [11, Corollary 3E]).

Within the more general framework of integration with respect to a vector measure, we see in Section 3 that simple functions are dense in the spaces of $S^{*}$ and McShane integrable functions (Theorems 3.3 and 3.5). The intimate relationship between the approximation by simple functions and the norm relative compactness of the indefinite integral will also be discussed.

Going back to the papers by Birkhoff and Pettis, one finds that both authors used the same sequence of simple functions to show that the spaces of Birkhoff and Pettis integrable functions from $[0,1]$ (with the Lebesgue measure) to $L^{2}[0,1]$ are not complete. More generally, Thomas [30] (and Janicka and Kalton [17] in the case of $[0,1])$ proved that the space of Pettis integrable functions defined on a non-purelyatomic probability space with values in an infinite dimensional Banach space is never complete. This result has been extended in [14, Theorem 8] to the space of Dobrakov integrable functions in the setting of injective tensor product integration (see case (C2) in Section 2). In view of the above, the spaces of $S^{*}$ and McShane integrable functions are not complete in general.

Fortunately, the space of Pettis integrable functions is always ultrabornological (Diaz et al., see [4, Theorem 2]), hence we can apply the closed graph theorem to linear mappings defined on it with values in the wide class of webbed locally convex spaces (that includes Fréchet spaces, the space of test functions and the space of distributions), see [18]. The same conclusion holds for the space of McShane integrable functions defined on [0, 1] (respectively $\mathbb{R}$ ), see [4, Theorem 3] (respectively [27, Corollary 15]).. In particular, these spaces are barrelled. For more examples of ultrabornological non complete normed spaces of vector-valued measurable and integrable functions we 
refer the reader to [4], [15, Chapter 4], [16] and [21].

The results in [4] are based on a general principle ([4, Corollary 1]) stating that a metrizable locally convex space with a suitable Boolean algebra of projections is ultrabornological. In Section 4, we successfully apply this criterion to the spaces of vector-valued functions that are Dobrakov, $S^{*}$ or McShane integrable with respect to a measure $\mu$ with values in the space of bounded operators between two Banach spaces. This is possible after some technical work that may be of interest in its own right and that extends to this more general setting some previous results spread out in the literature. As a consequence we will deduce that each one of these spaces is ultrabornological if and only if it is barrelled if and only if $\mu(A)$ is an operator with closed range for every atom $A$ of the semivariation of $\mu$ (Theorems 4.7, 4.13 and 4.20). We present examples showing that these spaces are neither ultrabornological nor barrelled in general, which invalidates a statement of Swartz [29, Theorem 16] regarding the barrelledness of the space of Dobrakov integrable functions. On the other hand, in the particular case of integration with respect to a scalar measure, our results apply to conclude that the spaces of vector-valued Birkhoff (respectively McShane) integrable functions defined on a complete (respectively quasi-Radon) probability space are ultrabornological (Corollaries 4.14 and 4.21).

\section{Preliminaries}

For the convenience of the reader this section is devoted to introduce the notation, definitions and preliminary results about the Dobrakov, $S^{*}$ and McShane integrals that are used throughout this paper.

2.1. Notation and terminology Our standard references are [22] (barrelled and ultrabornological locally convex spaces) and [5] (vector measures and integration of vector-valued functions).

All our vector spaces are assumed to be real. Given a Banach space $Z$, we denote by $B_{Z}$ the closed unit ball of $Z$, and $Z^{*}$ stands for the (topological) dual of $Z$. For a seminormed space $(F,\|\cdot\|)$, we denote by $\left(F^{*},\|\cdot\|\right)$ the corresponding normed space of equivalence classes obtained by the usual identification process, and we write $f^{*}$ to denote the class of each $f \in F$. A Hausdorff locally convex space $E$ is barrelled if every barrel in $E$ (that is, a closed, absolutely convex and absorbing subset of $E$ ) is a neighborhood of 0 . We say that $E$ is ultrabornological if every absolutely convex subset of $E$ that absorbs all Banach discs of $E$ is a neighborhood of 0 (equivalently, $E$ is an inductive limit of Banach spaces). Every ultrabornological locally convex space is barrelled, see for example [22, Section 6.1]. A projection on a vector space $W$ is a linear mapping $P: W \longrightarrow W$ such that $P \circ P=P$.

For a measurable space $(\Omega, \Sigma)$, a Banach space $Z$ and a countably additive vector 
measure $v: \Sigma \longrightarrow Z$, the scalar semivariation of $v[5$, page 2] is the function $\|v\|: \Sigma \longrightarrow[0, \infty)$ given by $\|v\|(E)=\sup _{z^{*} \in B_{z^{*}}}\left|z^{*} v\right|(E)$, where $\left|z^{*} v\right|$ denotes the variation of the signed measure $z^{*} v$. It is well known [5, Proposition 11, page 4] that for each $E \in \Sigma$,

$$
\sup \{\|v(A)\|: A \subset E, A \in \Sigma\} \leq\|v\|(E) \leq 2 \sup \{\|v(A)\|: A \subset E, A \in \Sigma\} .
$$

Let $\theta$ be a complete probability measure on $\Sigma$. For a Pettis $\theta$-integrable function $f: \Omega \longrightarrow Z$, we define $\|f\|_{P}:=\sup _{z^{*} \in B_{Z^{*}}} \int_{\Omega}\left|z^{*} f\right| d \theta<\infty$. Clearly $\|\cdot\|_{P}$ is a seminorm on the set of all Pettis $\theta$-integrable functions from $\Omega$ to $Z$ and the induced norm on the space of scalar equivalence classes is usually called the Pettis norm.

Throughout this paper $X$ and $Y$ are Banach spaces, $(\Omega, \Sigma)$ is a measurable space and $\mu: \Sigma \longrightarrow \mathcal{L}(X, Y)$ is a countably additive vector measure, where $\mathcal{L}(X, Y)$ is the Banach space of all bounded operators from $X$ to $Y$.

The semivariation of $\mu[6$, page 513] is the function $\hat{\mu}: \Sigma \rightarrow[0, \infty]$ defined by $\hat{\mu}(E)=\sup \left\|\sum_{i=1}^{n} \mu\left(E_{i}\right)\left(x_{i}\right)\right\|$, where the supremum is taken over all finite partitions $\left(E_{i}\right)_{i=1}^{n}$ of $E$ in $\Sigma$ and all finite collections $\left(x_{i}\right)_{i=1}^{n}$ in $B_{X}$. In addition, $\hat{\mu}$ is monotone and countably subadditive. Throughout this paper we will assume that $\hat{\mu}$ is continuous in the sense of [7, page 17]: for each decreasing sequence $\left(E_{n}\right)_{n=1}^{\infty}$ in $\Sigma$ such that $\bigcap_{n=1}^{\infty} E_{n}=\emptyset$, we have $\lim _{n} \hat{\mu}\left(E_{n}\right)=0$; equivalently, there is a non-negative finite measure $\lambda$ on $\Sigma$ such that $\lim _{\lambda(A) \rightarrow 0} \hat{\mu}(A)=0$ and $\lim _{\hat{\mu}(A) \rightarrow 0} \lambda(A)=0$, see [7, Lemma 2]. Throughout this paper $\lambda$ will always be such a measure. From the continuity of $\hat{\mu}$ it follows that $\hat{\mu}(\Omega)<\infty$ (see the remarks after Lemma 2 in [7]). Observe that for each $E \in \Sigma$ the restriction of $\mu$ to the $\sigma$-algebra $\Sigma_{E}=\{A \in \Sigma$ : $A \subset E\}$, denoted by $\mu_{E}$, is countably additive and has continuous semivariation. Clearly, the restriction of $\lambda$ to $\Sigma_{E}$, denoted by $\lambda_{E}$, fulfills $\lim _{\lambda_{E}(A) \rightarrow 0} \widehat{\mu_{E}}(A)=0$ and $\lim _{\widehat{\mu_{E}}(A) \rightarrow 0} \lambda_{E}(A)=0$.

A set $B \in \Sigma$ is an atom of $\hat{\mu}$ if $\hat{\mu}(B)>0$ and for each $A \in \Sigma_{B}$ either $\hat{\mu}(A)=0$ or $\hat{\mu}(B \backslash A)=0$ (equivalently, $B$ is an atom of $\lambda$ ). As usual, the outer measure induced by $\lambda$ will be denoted by $\lambda^{*}$.

We stress that $\hat{\mu}$ is continuous in the particular cases (C1) and (C2) isolated below. Further cases can be found in [1] and [20].

(C1) Integration of $X$-valued functions with respect to a non-negative finite measure $\nu$ on $\Sigma$. In such a case we take $Y:=X$ and $\mu(E)(x):=\nu(E) x$ for every $E \in \Sigma$ and every $x \in X$.

(C2) Injective tensor product integration of $X$-valued functions with respect to a countably additive vector measure $v: \Sigma \longrightarrow Z$, where $Z$ is another Banach space. In such a case we take $Y:=X \check{\otimes} Z$ (the injective tensor product of $X$ and $Z$, see [5, Chapter VIII]) and $\mu(E)(x):=x \otimes v(E)$ for every $E \in \Sigma$ and every $x \in X$. It can be easily checked that $\|v\|=\hat{\mu}$. By [5, Corollary 6, page 14], there is a non-negative 
finite measure $\lambda$ on $\Sigma$ such that $\lim _{\lambda(A) \rightarrow 0}\|\nu\|(A)^{-}=0$ and $\lim _{\|\nu\|(A) \rightarrow 0} \lambda(A)=0$, hence $\hat{\mu}$ is continuous. Clearly, case (C2) includes (C1).

\subsection{The Dobrakov, $\boldsymbol{S}^{*}$ and McShane integrals A function $f: \Omega \longrightarrow X$ of} the form $f=\sum_{i=1}^{n} x_{i} \chi_{A_{i}}, x_{i} \in X, A_{i} \in \Sigma$, is called simple. In this case we write $\int_{\Omega} f d \mu:=\sum_{i=1}^{n} \mu\left(A_{i}\right)\left(x_{i}\right)$. The family of all simple functions from $\Omega$ to $X$ will be denoted by $\mathcal{S}(\Sigma, X)$.

A function $f: \Omega \longrightarrow X$ is called measurable if there is a sequence in $\mathcal{S}(\Sigma, X)$ converging pointwise to $f$. A function $f: \Omega \longrightarrow X$ is Dobrakov integrable with respect to $\mu$, see [6, Definition 2 and Theorem 7], if it is measurable and there is a sequence $\left(f_{n}\right)$ in $\mathcal{S}(\Sigma, X)$ converging to $f \hat{\mu}$-almost everywhere such that for every $E \in \Sigma$ there exists $\lim _{n} \int_{E} f_{n} d \mu_{E}$, for the norm topology of $Y$. The Dobrakov integral of $f$ with respect to $\mu$ is defined by

$$
\text { (D) } \int_{\Omega} f d \mu=\lim _{n} \int_{\Omega} f_{n} d \mu \text {. }
$$

The family of all functions from $\Omega$ to $X$ that are Dobrakov integrable with respect to $\mu$ will be denoted by $D(\mu)$. It is easy to check that $D(\mu)$ is a linear subspace of $X^{\Omega}$ and that the map from $D(\mu)$ to $Y$ given by $f \mapsto(D) \int_{\Omega} f d \mu$ is linear. Observe also that for each $f \in D(\mu)$ and each $A \in \Sigma$ the restriction $\left.f\right|_{A}$ is Dobrakov integrable wiih respect to $\mu_{A}$. Moreover, the indefinite integral $I_{f}: \Sigma \longrightarrow Y$ given by $I_{f}(A):=(D) \int_{A} f d \mu_{A}$ is a countably additive vector measure, see [6, Theorem 3]. Throughout this paper we consider the space $D(\mu)$ endowed with the seminorm $\|\cdot\|_{I}$ defined by $\|f\|_{I}:=\left\|I_{f}\right\|(\Omega)$.

Given a function $f: \Omega \longrightarrow X$, a countable family $\Gamma=\left(A_{n}\right)$ of pairwise disjoint elements of $\Sigma$ and a choice $T=\left(t_{n}\right)$ in $\Gamma$ (that is, $t_{n} \in A_{n}$ for every $n$ ), the symbol

$$
S(f, \Gamma, T):=\sum_{n} \mu\left(A_{n}\right)\left(f\left(t_{n}\right)\right)
$$

denotes a formal series in $Y$. As usual, we say that another countable family $\Gamma^{\prime}$ of pairwise disjoint elements of $\Sigma$ is finer than $\Gamma$ provided that each element of $\Gamma^{\prime}$ is contained in some element of $\Gamma$.

A function $f: \Omega \longrightarrow X$ is $S^{*}$-integrable with respect to $\mu$, with $S^{*}$-integral $y \in Y$, see [8, Definition 1], if for every $\epsilon>0$ there is a countable partition $\Gamma_{0}$ of $\Omega$ in $\Sigma$ such that for every countable partition $\Gamma$ of $\Omega$ in $\Sigma$ finer than $\Gamma_{0}$ and every choice $T$ in $\Gamma$, the series $S(f, \Gamma, T)$ is unconditionally convergent in $Y$ and $\|S(f, \Gamma, T)-y\| \leq \epsilon$. The vector $y$ (necessarily unique) is denoted by $\left(S^{*}\right) \int_{\Omega} f d \mu$. The family of all functions from $\Omega$ to $X$ which are $S^{*}$-integrable with respect to $\mu$ is a linear subspace of $X^{\Omega}$ that we denote by $S^{*}(\mu)$. Clearly, the map from $S^{*}(\mu)$ to $Y$ given by $f \mapsto\left(S^{*}\right) \int_{\Omega} f d \mu$ is linear. For each $f \in S^{*}(\mu)$ we know that: 
(i) $\left.f\right|_{A}$ is $S^{*}$-integrable with respect to $\mu_{A}$ for every $A \in \Sigma$; (ii) the indefinite integral $v_{f}: \Sigma \longrightarrow Y$ given by $v_{f}(A):=\left(S^{*}\right) \int_{A} f d \mu_{A}$ is a countably additive vector measure, see [8, Lemma 1 (1)]. Throughout we consider the space $S^{*}(\mu)$ equipped with the seminorm $\|\cdot\|_{\nu}$ given by $\|f\|_{\nu}:=\left\|v_{f}\right\|(\Omega)$.

We stress that a function $f: \Omega \longrightarrow X$ is Dobrakov integrable with respect to $\mu$ if and only if $f$ is measurable and $S^{*}$-integrable with respect to $\mu$ (in this case, $I_{f}=v_{f}$ ), see [8, Theorem 1]. Therefore, $\left(D(\mu),\|\cdot\|_{I}\right)$ is a subspace of $\left(S^{*}(\mu),\|\cdot\|_{\nu}\right)$.

Throughout Subsections 3.2 and 4.3 we consider the McShane integral with respect to a vector measure. As in the case of scalar measures, further conditions are required to set up this integral. We assume that $\tau$ is a topology on $\Omega$ with $\tau \subset \Sigma$ and we suppose that $(\Omega, \tau, \Sigma, \lambda)$ is a finite quasi-Radon measure space, in the sense of [12, Section 411H] (for instance, a finite Radon measure space, see [12, Section 416A]). Equivalently, we will assume that $\hat{\mu}$ satisfies the following properties:

$(\alpha)$ for every $E \in \Sigma$ and every $\epsilon>0$ there exists a $\tau$-closed set $C \subset E$ such that $\hat{\mu}(E \backslash C) \leq \epsilon$

$(\beta) \inf _{G \in \mathcal{G}} \hat{\mu}(\cup \mathcal{G} \backslash G)=0$ for every non-empty upwards directed family $\mathcal{G}$ of $\tau$-open sets;

( $\gamma)$ if $A \subset E \in \Sigma$ and $\hat{\mu}(E)=0$, then $A \in \Sigma$.

For each $A \in \Sigma$ the set function $\widehat{\mu_{A}}$ fulfills conditions $(\alpha),(\beta)$ and $(\gamma)$ with respect to $\Sigma_{A}$ and the induced topology $\tau_{A}=\{B \cap A: B \in \tau\}$, since $\left(A, \tau_{A}, \Sigma_{A}, \lambda_{A}\right)$ is a finite quasi-Radon measure space too, see [12, Section 415B]. For some examples of topological spaces and vector measures satisfying the conditions above we refer the reader to [26].

To recall the definition of the McShane integral we need some terminology. A gauge on $(\Omega, \tau)$ is a function $\delta: \Omega \longrightarrow \tau$ such that $\omega \in \delta(\omega)$ for every $\omega \in \Omega$. A partial McShane partition of $\Omega$ is a finite collection $\mathcal{P}=\left\{\left(E_{i}, s_{i}\right): 1 \leq i \leq p\right\}$ where $\left(E_{i}\right)_{i=1}^{p}$ are pairwise disjoint elements of $\Sigma$ and $s_{i} \in \Omega$ for every $1 \leq i \leq p$. We write $W_{\mathcal{P}}:=\bigcup_{i=1}^{p} E_{i}$. The collection $\mathcal{P}$ is said to be subordinate to $\delta$ if $E_{i} \subset \delta\left(s_{i}\right)$ for every $1 \leq i \leq p$. For every gauge $\delta$ on $(\Omega, \tau)$ and every $\eta>0$, we write $\Pi_{\delta, \eta}$ to denote the set of all partial McShane partitions $\mathcal{P}$ of $\Omega$ subordinate to $\delta$ such that $\hat{\mu}\left(\Omega \backslash W_{\mathcal{P}}\right) \leq \eta$. Since $(\Omega, \tau, \Sigma, \lambda)$ is a finite quasi-Radon measure space, the arguments in $[11$, Section $1 \mathrm{~B}(\mathrm{~d})]$ show that $\Pi_{\delta, \eta} \neq \emptyset$.

In the sequel, given a function $f: \Omega \longrightarrow X$ and a partial McShane partition $\mathcal{P}=\left\{\left(E_{i}, s_{i}\right): 1 \leq i \leq p\right\}$ of $\Omega$, we write $f(\mathcal{P}):=\sum_{i=1}^{p} \mu\left(E_{i}\right)\left(f\left(s_{i}\right)\right) \in Y$.

A function $f: \Omega \longrightarrow X$ is McShane integrable with respect to $\mu$, with McShane integral $y \in Y$, see [26, Definition 3.1], if for every $\epsilon>0$ there exist a gauge $\delta$ on $(\Omega, \tau)$ and $\eta>0$ such that $\|f(\mathcal{P})-y\| \leq \epsilon$ for every $\mathcal{P} \in \Pi_{\delta, \eta}$. The vector $y \in Y$ (necessarily unique) is denoted by $(M) \int_{\Omega} f d \mu$. The family of all functions from $\Omega$ to $X$ which are McShane integrable with respect to $\mu$ is a linear subspace of $X^{\Omega}$ that 
will be denoted by $M(\mu)$. The map from $M(\mu)$ to $Y$ given by $f \mapsto(M) \int_{\Omega} f d \mu$ is linear. As for the Dobrakov and $S^{*}$ integrals, if $f \in M(\mu)$, then (i) $\left.f\right|_{A}$ is McShane integrable with respect to $\mu_{A}$ for every $A \in \Sigma$; (ii) the set function $\zeta_{f}: \Sigma \longrightarrow Y$ given by $\zeta_{f}(A):=(M) \int_{A} f d \mu_{A}$ is a countably additive vector measure, see [26, Lemma 3.2]. From now on we consider the space $M(\mu)$ endowed with the seminorm $\|\cdot\|_{\zeta}$ defined by $\|f\|_{\zeta}:=\left\|\zeta_{f}\right\|(\Omega)$.

As we mentioned in the introduction, our paper [26] deals with the relationship between the McShane, $S^{*}$ and Dobrakov integrals. For a function $f: \Omega \longrightarrow X$, we know that

(a) if $f$ is $S^{*}$-integrable with respect to $\mu$, then $f$ is McShane integrable with respect to $\mu$ and $v_{f}=\zeta_{f}$, see [26, Theorem 3.7];

(b) $f$ is Dobrakov integrable with respect to $\mu$ if and only if $f$ is measurable and McShane integrable with respect to $\mu$, see [26, Theorem 3.8].

From (a) it follows that $\left(S^{*}(\mu),\|\cdot\|_{\nu}\right)$ is a subspace of $\left(M(\mu),\|\cdot\|_{\zeta}\right)$.

Finally, we remark that in the particular case of integration of vector-valued functions defined on a probability space (see case (C1) in Subsection 2.1), the notions of Dobrakov, $S^{*}$ and McShane integrability correspond to the notions of Pettis (for measurable functions), Birkhoff and generalized McShane integrability, respectively, see [1, 6], [3, Proposition 2.6] and [10, Proposition 3].

\section{Density of simple functions in the spaces of $S^{*}$ and McShane integrable functions}

It is known that the space $\mathcal{S}(\Sigma, X)$ of all $X$-valued simple functions defined on $\Omega$ is a dense subspace of $\left(D(\mu),\|\cdot\|_{I}\right)$, see [6, Theorem 7]. The aim of this section is to extend this result to the spaces $\left(S^{*}(\mu),\|\cdot\|_{\nu}\right)$ and $\left(M(\mu),\|\cdot\|_{\zeta}\right)$. As an application we discuss the norm relative compactness of the range of the indefinite integral $v_{f}$ (respectively $\zeta_{f}$ ) associated to an $S^{*}$-integrable (respectively McShane integrable) function $f$.

3.1. Spaces of $S^{*}$-integrable functions The following elementary lemmas will be needed in the proofs of Theorem 3.3 below and some of the results stated in Subsection 4.2. We refer the reader to [26] for the details.

LEMMA 3.1 ([26, Lemma 2.1]). Let $f \in S^{*}(\mu)$. Then for each $\epsilon>0$ there is a countable partition $\Gamma_{0}$ of $\Omega$ in $\Sigma$ such that for every countable family $\Gamma=\left(A_{n}\right)$ of pairwise disjoint elements of $\Sigma$ finer than $\Gamma_{0}$ and every choice $T$ in $\Gamma$, the series $S(f, \Gamma, T)$ is unconditionally convergent and $\left\|S(f, \Gamma, T)-v_{f}\left(\bigcup_{n} A_{n}\right)\right\| \leq \epsilon$. 
LEMMA 3.2 ([26, Lemma 2.2]). Suppose that $\Omega$ is an atom of $\hat{\mu}$. If $f \in S^{*}(\mu)$, then there is $E \in \Sigma$ such that $\hat{\mu}(\Omega \backslash E)=0$ and $v_{f}(\Omega)=\mu(\Omega)(f(\omega))$ for every $\omega \in E$.

THEOREM 3.3. $\mathcal{S}(\Sigma, X)$ is dense in $\left(S^{*}(\mu),\|\cdot\|_{v}\right)$.

ProOF. We have to check that for every $f \in S^{*}(\mu)$ and every $\epsilon>0$ there is a simple function $g: \Omega \longrightarrow X$ such that $\sup _{E \in \Sigma}\left\|v_{f}(E)-v_{g}(E)\right\| \leq \epsilon$. For the proof we distinguish two cases.

Particular case. Suppose that $\lambda$ is atomless. Lemma 3.1 implies that there is a countable partition $\Gamma_{0}=\left(A_{n}\right)$ of $\Omega$ in $\Sigma$ such that

$$
\left\|S(f, \Gamma, T)-v_{f}\left(\bigcup_{m} B_{m}\right)\right\| \leq \frac{\epsilon}{2}
$$

for every countable family $\Gamma=\left(B_{m}\right)$ of pairwise disjoint elements of $\Sigma$ finer than $\Gamma_{0}$ and every choice $T$ in $\Gamma$, the series $S(f, \Gamma, T)$ being unconditionally convergent.

Since $v_{f}$ is countably additive and $v_{f}(E)=0$ for every $E \in \Sigma$ with $\lambda(E)=0$, we have

$$
\lim _{\lambda(E) \rightarrow 0} v_{f}(E)=0
$$

see [5, Theorem 1, page 10]. Hence we can choose $N \in \mathbb{N}$ large enough such that $\left\|v_{f}(B)\right\| \leq \epsilon / 2$ for every $B \subset \bigcup_{n>N} A_{n}, B \in \Sigma$.

Fix $\omega_{n} \in A_{n}$ for every $1 \leq n \leq N$ and define $g:=\sum_{n=1}^{N} f\left(\omega_{n}\right) \chi_{A_{n}} \in \mathcal{S}(\Sigma, X)$. We claim that

$$
\sup _{E \in \Sigma}\left\|v_{f}(E)-v_{g}(E)\right\| \leq \epsilon .
$$

Indeed, take any $E \in \Sigma$ and fix $\eta>0$. Since $\lambda$ is atomless, $\lambda^{*}\left(\left\{\omega_{n}\right\}\right)=0$ for every $1 \leq n \leq N$. Therefore, bearing in mind (3.2), for each $1 \leq n \leq N$ we can choose $E_{n} \subset A_{n}, \omega_{n} \in E_{n} \in \Sigma$, such that

$$
\max _{1 \leq n \leq N}\left\|f\left(\omega_{n}\right)\right\| \hat{\mu}\left(\bigcup_{n=1}^{N}\left(E_{n} \backslash E\right)\right) \leq \eta \quad \text { and }\left\|v_{f}\left(\bigcup_{n=1}^{N}\left(E_{n} \backslash E\right)\right)\right\| \leq \eta .
$$

Since $\Gamma=\left\{\left(E \cap A_{n}\right) \cup E_{n}: 1 \leq n \leq N\right\}$ is a finite collection of pairwise disjoint elements of $\Sigma$ finer than $\Gamma_{0}$, (3.1) yields

$$
\left\|\sum_{n=1}^{N} \mu\left(\left(E \cap A_{n}\right) \cup E_{n}\right)\left(f\left(\omega_{n}\right)\right)-v_{f}\left(\bigcup_{n=1}^{N}\left(\left(E \cap A_{n}\right) \cup E_{n}\right)\right)\right\| \leq \frac{\epsilon}{2} .
$$


This inequality and (3.4) yield

$$
\begin{aligned}
&\left\|\sum_{n=1}^{N} \mu\left(E \cap A_{n}\right)\left(f\left(\omega_{n}\right)\right)-v_{f}\left(\bigcup_{n=1}^{N}\left(E \cap A_{n}\right)\right)\right\| \\
& \leq\left\|\sum_{n=1}^{N} \mu\left(\left(E \cap A_{n}\right) \cup E_{n}\right)\left(f\left(\omega_{n}\right)\right)-v_{f}\left(\bigcup_{n=1}^{N}\left(\left(E \cap A_{n}\right) \cup E_{n}\right)\right)\right\| \\
&+\left\|\sum_{n=1}^{N} \mu\left(E_{n} \backslash E\right)\left(f\left(\omega_{n}\right)\right)\right\|+\left\|v_{f}\left(\bigcup_{n=1}^{N}\left(E_{n} \backslash E\right)\right)\right\| \leq \frac{\epsilon}{2}+2 \eta .
\end{aligned}
$$

The fact that $\nu_{g}(E)=\sum_{n=1}^{N} \mu\left(E \cap A_{n}\right)\left(f\left(\omega_{n}\right)\right)$ and the choice of $N$ imply

$$
\begin{aligned}
\left\|v_{g}(E)-v_{f}(E)\right\| & \leq\left\|v_{g}(E)-v_{f}\left(\bigcup_{n=1}^{N}\left(E \cap A_{n}\right)\right)\right\|+\left\|v_{f}\left(\bigcup_{n>N}\left(E \cap A_{n}\right)\right)\right\| \\
& \leq \epsilon+2 \eta .
\end{aligned}
$$

As $E \in \Sigma$ and $\eta>0$ are arbitrary, (3.3) holds and the proof of the Particular case is complete.

General case. There is a (maybe empty) countable collection $\left(B_{n}\right)$ of pairwise disjoint atoms of $\lambda$ such that, if we write $A=\Omega \backslash \bigcup_{n} B_{n}$, then $\lambda_{A}$ is atomless.

Since $\left.f\right|_{A} \in S^{*}\left(\mu_{A}\right)$, the Particular case already proved ensures us that there is a simple function $h: A \longrightarrow X$ such that

$$
\sup _{E \in \Sigma_{A}}\left\|v_{f}(E)-v_{h}(E)\right\| \leq \frac{\epsilon}{2}
$$

As in the proof of the Particular case, we can choose $N \in \mathbb{N}$ large enough such that $\left\|v_{f}(B)\right\| \leq \epsilon / 2$ for every $B \subset \bigcup_{n>N} B_{n}, B \in \Sigma$. According to Lemma 3.2, applied to each $\left.f\right|_{B_{n}}$, for every $1 \leq n \leq N$ there exists $\omega_{n} \in B_{n}$ such that

$$
\nu_{f}(E)=\mu(E)\left(f\left(\omega_{n}\right)\right) \text { for every } E \in \Sigma_{B_{n}} .
$$

Let us consider the simple function $g: \Omega \longrightarrow X$ defined by

$$
g(\omega)= \begin{cases}h(\omega) & \text { if } \omega \in A \\ f\left(\omega_{n}\right) & \text { if } \omega \in B_{n} \text { for some } 1 \leq n \leq N \\ 0 & \text { otherwise. }\end{cases}
$$


We claim that $\sup _{E \in \Sigma}\left\|v_{f}(E)-v_{g}(E)\right\| \leq \epsilon$. Indeed, given $E \in \Sigma$, we have

$$
\begin{aligned}
\left\|v_{f}(E)-v_{g}(E)\right\| \leq & \left\|v_{f}(E \cap A)-v_{h}(E \cap A)\right\| \\
& +\left\|\sum_{n=1}^{N}\left(v_{f}\left(E \cap B_{n}\right)-v_{g}\left(E \cap B_{n}\right)\right)\right\|+\left\|v_{f}\left(\bigcup_{n>N}\left(E \cap B_{n}\right)\right)\right\| \\
\leq & \frac{\epsilon}{2}+\frac{\epsilon}{2}=\epsilon,
\end{aligned}
$$

by (3.5), (3.6) and the choice of $N$. This finishes the proof of the theorem.

COROLLARY 3.4. The following conditions are equivalent:

(i) $\{\mu(E)(x): E \in \Sigma\}$ is norm relatively compact for every $x \in X$;

(ii) $\left\{v_{f}(A): A \in \Sigma\right\}$ is norm relatively compact for every $f \in S^{*}(\mu)$.

Proof. (i) implies (ii). Fix $f \in S^{*}(\mu)$. It suffices to check that $\left\{\nu_{f}(A): A \in \Sigma\right\}$ is totally bounded by the norm. Fix $\epsilon>0$. By Theorem 3.3 there is a simple function $g=\sum_{i=1}^{n} x_{i} \chi_{A_{i}}, x_{i} \in X, A_{i} \in \Sigma$, such that

$$
\sup _{E \in \Sigma}\left\|v_{f}(E)-v_{g}(E)\right\|<\epsilon \text {. }
$$

Since for each $1 \leq i \leq n$ the set $\overline{\left\{\mu(E)\left(x_{i}\right): E \in \Sigma\right\}^{n \cdot h}}$ is norm compact and

$$
\left\{\nu_{g}(E): E \in \Sigma\right\} \subset \sum_{i=1}^{n} \overline{\left\{\mu(E)\left(x_{i}\right): E \in \Sigma\right\}}\|\cdot\|,
$$

there are $E_{1}, \ldots, E_{k} \in \Sigma$ such that $\inf _{1 \leq i \leq k}\left\|v_{g}(E)-v_{g}\left(E_{i}\right)\right\|<\epsilon$ for every $E \in \Sigma$. This inequality and (3.7) yield $\inf _{1 \leq i \leq k}\left\|v_{f}(E)-v_{f}\left(E_{i}\right)\right\|<3 \epsilon$ for every $E \in \Sigma$. As $\epsilon>0$ is arbitrary, $\left\{v_{f}(A): A \in \Sigma\right\}$ is norm relatively compact and the statement (i) implies (ii) has been established.

Conversely, assume that (ii) holds, fix $x \in X$ and define $f:=x \chi_{\Omega} \in S^{*}(\mu)$. Then $\left\{v_{f}(A): A \in \Sigma\right\}=\{\mu(E)(x): E \in \Sigma\}$ is norm relatively compact. The proof is complete.

Corollary 3.4 generalizes the fact that the range of the indefinite integral of a Birkhoff integrable function is norm relatively compact, see [2, Theorem 18] and [3, Corollary 3.6]. Our approach here avoids the operator-theoretic arguments involving the Bourgain property used in [3] and it is closer to Birkhoff's ideas.

3.2. Spaces of McShane integrable functions As pointed out in the introduction, every McShane integrable vector-valued function defined on a quasi-Radon probability space is the limit, for the Pettis norm, of a sequence of simple functions. We next 
show that this result can be extended to the more general setting of this paper. Our proof does not involve the techniques about stable families of measurable functions that were used by Fremlin in the case of scalar measures. Throughout this subsection $\tau$ is a topology on $\Omega$ with $\tau \subset \Sigma$ and we assume that $\hat{\mu}$ satisfies properties $(\alpha),(\beta)$ and $(\gamma)$ mentioned in Subsection 2.2.

THEOREM 3.5. $\mathcal{S}(\Sigma, X)$ is dense in $\left(M(\mu),\|\cdot\|_{\zeta}\right)$.

ProOF. Fix $f \in M(\mu)$. We have to prove that for each $\epsilon>0$ there is $g \in \mathcal{S}(\Sigma, X)$ such that $\sup _{E \in \Sigma}\left\|\zeta_{f}(E)-\zeta_{g}(E)\right\| \leq \epsilon$.

By the Henstock-Saks Lemma [26, Lemma 3.3], there is a gauge $\delta$ on $(\Omega, \tau)$ such that

$$
\left\|\sum_{i=1}^{q} \mu\left(F_{i}\right)\left(f\left(t_{i}\right)\right)-\zeta_{f}\left(\bigcup_{i=1}^{q} F_{i}\right)\right\| \leq \frac{\epsilon}{2}
$$

for every partial McShane partition $\mathcal{P}=\left\{\left(F_{i}, t_{i}\right): 1 \leq i \leq q\right\}$ of $\Omega$ subordinate to $\delta$. Since $\lim _{\hat{\mu}(E) \rightarrow 0} \zeta_{f}(E)=0$, see [26, Lemma 3.2], there is an $\eta>0$ such that

$$
\left\|\zeta_{f}(B)\right\| \leq \epsilon / 2 \text { for every } B \in \Sigma \text { with } \hat{\mu}(B) \leq \eta .
$$

Fix a partial McShane partition $\left\{\left(E_{i}, s_{i}\right): 1 \leq i \leq p\right\}$ of $\Omega$ subordinate to $\delta$ such that $\hat{\mu}\left(\Omega \backslash \bigcup_{i=1}^{p} E_{i}\right) \leq \eta$. Let us define $g:=\sum_{i=1}^{p} f\left(s_{i}\right) \chi_{E_{i}} \in \mathcal{S}(\Sigma, X)$. We claim that

$$
\sup _{E \in \Sigma}\left\|\zeta_{f}(E)-\zeta_{g}(E)\right\| \leq \epsilon .
$$

Indeed, given $E \in \Sigma$, the collection $\left\{\left(E_{i} \cap E, s_{i}\right): 1 \leq i \leq p\right\}$ is a partial McShane partition of $\Omega$ subordinate to $\delta$, and (3.8) yields

$$
\left\|\sum_{i=1}^{p} \mu\left(E_{i} \cap E\right)\left(f\left(s_{i}\right)\right)-\zeta_{f}\left(E \cap\left(\bigcup_{i=1}^{p} E_{i}\right)\right)\right\| \leq \frac{\epsilon}{2} .
$$

Therefore

$$
\begin{aligned}
& \left\|\sum_{i=1}^{p} \mu\left(E_{i} \cap E\right)\left(f\left(s_{i}\right)\right)-\zeta_{f}(E)\right\| \\
& \quad \leq\left\|\sum_{i=1}^{p} \mu\left(E_{i} \cap E\right)\left(f\left(s_{i}\right)\right)-\zeta_{f}\left(E \cap\left(\bigcup_{i=1}^{p} E_{i}\right)\right)\right\|+\left\|\zeta_{f}\left(E \backslash\left(\bigcup_{i=1}^{p} E_{i}\right)\right)\right\| \\
& \quad \leq \epsilon,
\end{aligned}
$$

by (3.9). Since $\sum_{i=1}^{p} \mu\left(E_{i} \cap E\right)\left(f\left(s_{i}\right)\right)=\zeta_{g}(E)$, we get $\left\|\zeta_{f}(E)-\zeta_{g}(E)\right\| \leq \epsilon$. As $E \in \Sigma$ is arbitrary, (3.10) holds and the proof is finished. 
We can now proceed analogously to the proof of Corollary 3.4 to deduce the following result.

COROLLARY 3.6. The following conditions are equivalent:

(i) $\{\mu(E)(x): E \in \Sigma\}$ is norm relatively compact for every $x \in X$;

(ii) $\left\{\zeta_{f}(A): A \in \Sigma\right\}$ is norm relatively compact for every $f \in M(\mu)$.

\section{Ultrabornological spaces of integrable functions}

This section is devoted to characterizing when $D(\mu)^{\bullet}, S^{*}(\mu)^{\bullet}$ and $M(\mu)^{\bullet}$ are ultrabornological normed spaces. The criterion obtained in [4, Corollary 1] (see Proposition 4.2 below) plays an essential role in our analysis. In order to recall it (in the context of normed spaces) we first need a definition.

DEFINITION 4.1 ([4]). Let $(T, \mathcal{A}, \theta)$ be a finite measure space and $E$ a locally convex space. A $(T, \mathcal{A}, \theta)$-Boolean algebra of projections in $E$ is a family $\left\{P_{A}: A \in \mathcal{A}\right\}$ of continuous projections in $E$ such that

(i) $P_{\Omega}$ is the identity on $E$;

(ii) $P_{A \cap B}=P_{A} \circ P_{B}$ for every $A, B \in \mathcal{A}$;

(iii) $P_{A \cup B}=P_{A}+P_{B}$ for every $A, B \in \mathcal{A}$ with $A \cap B=\emptyset$;

(iv) $P_{A}=0$ whenever $\theta(A)=0$.

PROPOSITION 4.2 ([4, Corollary 1]). Let $(T, \mathcal{A}, \theta)$ be a finite measure space and $E$ a normed space. Suppose that there is an equicontinuous $(T, \mathcal{A}, \theta)$-Boolean algebra of projections $\left\{P_{A}: A \in \mathcal{A}\right\}$ in $E$ with the following properties:

(i) if $\left(B_{n}\right)_{n=1}^{\infty}$ is a decreasing sequence in $\mathcal{A}$ such that $\theta\left(\bigcap_{n=1}^{\infty} B_{n}\right)=0,\left(e_{n}\right)_{n=1}^{\infty}$ is a bounded sequence in $E$ such that $P_{B_{n}}\left(e_{n}\right)=e_{n}$ for every $n \in \mathbb{N}$, and $\left(\beta_{n}\right)_{n=1}^{\infty} \in \ell^{1}$, then the series $\sum_{n=1}^{\infty} \beta_{n} e_{n}$ converges in $E$;

(ii) $P_{A}(E)$ is ultrabornological for every atom $A$ of $\theta$.

Then $E$ is ultrabornological.

The result isolated in Corollary 4.4 below is an immediate consequence of Proposition 4.2 that will be applied to each one of the spaces of integrable functions that we consider in this paper. All of them are included in the following class of seminormed spaces.

DEFINITION 4.3. A seminormed space $(F,\|\cdot\|)$ belongs to the class $\mathfrak{J}_{\mu}$ if

(i) $F$ is a linear subspace of $X^{\Omega}$;

(ii) $f \chi_{A} \in F$ for every $f \in F$ and every $A \in \Sigma$; 
(iii) for each $f \in F$ there is a countably additive vector measure $V_{f}^{F}: \Sigma \longrightarrow Y$ such that

- $V_{f X_{A}}^{F}(\Omega)=V_{f}^{F}(A)$ for every $A \in \Sigma$;

- $V_{f}^{F}(E)=0$ whenever $\hat{\mu}(E)=0$;

- $\|f\|=\left\|V_{f}^{F}\right\|(\Omega)$;

(iv) $V_{\alpha f+g}^{F}=\alpha V_{f}^{F}+V_{g}^{F}$ for every $f, g \in F$ and every $\alpha \in \mathbb{R}$.

As usual, given a function $f: \Omega \longrightarrow X$ and $A \in \Sigma$, we denote by $f \chi_{A}$ the function from $\Omega$ to $X$ defined by $f \chi_{A}(\omega)=f(\omega)$ if $\omega \in A$, and $f \chi_{A}=0$ if $\omega \in \Omega \backslash A$.

Given a seminormed space $(F,\|\cdot\|)$ in the class $\mathfrak{J}_{\mu}$, it is clear that $V_{f X_{A}}^{F}(B)=$ $V_{f}^{F}(A \cap B)$ for every $A, B \in \Sigma$. Thus for each $A \in \Sigma$ we can define a mapping $P_{A}^{F}: F^{*} \longrightarrow F^{*}$ by $P_{A}^{F}\left(f^{*}\right):=\left(f \chi_{A}\right)^{*}$. It is easy to see that $\left\{P_{A}^{F}: A \in \Sigma\right\}$ is an equicontinuous $(\Omega, \Sigma, \lambda)$-Boolean algebra of projections on $\left(F^{*},\|\cdot\|\right)$. We write $P_{A}$ instead of $P_{A}^{F}$ and $V_{f}$ instead of $V_{f}^{F}$ when no confusion is possible.

COROLLARY 4.4. Let $(F,\|\cdot\|)$ be a seminormed space in the class $\mathfrak{J}_{\mu}$ with the following properties:

(i) if $\left(A_{n}\right)_{n=1}^{\infty}$ is a countable partition of $\Omega$ in $\Sigma,\left(f_{n}\right)_{n=1}^{\infty}$ is a bounded sequence in $(F,\|\cdot\|)$ such that $\left.f_{n}\right|_{A_{m}} \equiv 0$ whenever $n>m$, and $\left(\alpha_{n}\right)_{n=1}^{\infty} \in \ell^{1}$, then the function $f: \Omega \longrightarrow X$ given by $f:=\sum_{n=1}^{\infty} \alpha_{n} f_{n}$ belongs to $F$ and $\lim _{N}\left\|\sum_{n=1}^{N} \alpha_{n} f_{n}-f\right\|=0$;

(ii) $P_{A}\left(F^{\circ}\right)$ is ultrabornological for every atom $A$ of $\hat{\mu}$.

Then $\left(F^{\cdot},\|\cdot\|\right)$ is ultrabornological.

ProOF. In view of Proposition 4.2, it suffices to check condition (i) of that result. To this end, we fix a decreasing sequence $\left(B_{n}\right)_{n=1}^{\infty}$ in $\Sigma$ such that $\lambda\left(\bigcap_{n=1}^{\infty} B_{n}\right)=0$, a bounded sequence $\left(h_{n}^{*}\right)_{n=1}^{\infty}$ in $F^{\cdot}$ such that $P_{B_{n}}\left(h_{n}^{*}\right)=h_{n}^{*}$ for every $n \in \mathbb{N}$, and $\left(\beta_{n}\right)_{n=1}^{\infty} \in \ell^{1}$. Define $A_{1}:=\bigcap_{n=1}^{\infty} B_{n}, A_{2}:=\Omega \backslash B_{1}$ and $A_{n}:=B_{n-2} \backslash B_{n-1}$ for every $n \geq 3$. Then $\left(A_{n}\right)_{n=1}^{\infty}$ is a partition of $\Omega$ in $\Sigma$. Since $\lambda\left(A_{1}\right)=0$, we can suppose without loss of generality that $\left.h_{n}\right|_{A_{1}} \equiv 0$ for every $n \in \mathbb{N}$. Define $f_{1}=$ $f_{2}=0 \in F$ and $f_{n}:=h_{n-2} \chi_{B_{n-2}} \in F$ for every $n \geq 3$. Clearly $\left.f_{n}\right|_{A_{m}} \equiv 0$ whenever $n>m$. Notice that $f_{n}^{\cdot}=\left(h_{n-2} \chi_{B_{n-2}}\right)^{\bullet}=P_{B_{n-2}}\left(h_{n-2}^{\cdot}\right)=h_{n-2}^{\cdot}$ for every $n \geq 3$, hence $\left(f_{n}\right)_{n=1}^{\infty}$ is a bounded sequence in $F$ and (i) yields that $f:=\sum_{n=3}^{\infty} \beta_{n-2} f_{n} \in F$ and $\lim _{N}\left\|\sum_{n=3}^{N} \beta_{n-2} f_{n}-f\right\|=0$. Accordingly, $\sum_{n=1}^{\infty} \beta_{n} h_{n}^{*}$ converges in $\left(F^{*},\|\cdot\|\right)$ and the proof is complete.

As regards to condition (ii) in Corollary 4.4 , the following result clarifies the differences between the case of integration with respect to a non-negative finite measure and the general case.

PROPOSITION 4.5. Let $(F,\|\cdot\|)$ be a seminormed space in the class $\mathfrak{J}_{\mu}$ such that $\mathcal{S}(\Sigma, X) \subset F$ and $V_{g}(\Omega)=\int_{\Omega} g d \mu$ for every $g \in \mathcal{S}(\Sigma, X)$. Suppose that $A \in \Sigma$ is 
an atom of $\hat{\mu}$ such that $V_{f}(A) \in \mu(A)(X)$ for every $f \in F$. Then $P_{A}\left(F^{*}\right)=\left\{\left(x \chi_{A}\right)^{\bullet}\right.$ : $x \in X\}, P_{A}\left(F^{*}\right)$ is linearly homeomorphic to $\mu(A)(X)$, and the following conditions are equivalent:

(i) $P_{A}\left(F^{*}\right)$ is a Banach space;

(ii) $P_{A}\left(F^{*}\right)$ is ultrabornological;

(iii) $P_{A}\left(F^{*}\right)$ is barrelled;

(iv) $\mu(A)(X)$ is closed in $Y$.

Proof. Write $T:=\mu(A)$ and $\operatorname{ker} T=\{x \in X: T(x)=0\}$. We denote by $\tilde{T}$ the induced continuous linear bijection from the quotient Banach space $X / \operatorname{ker} T$ onto $T(X)$ defined by $\tilde{T}(x+\operatorname{ker} T)=T(x)$. Clearly, the inverse $\tilde{T}^{-1}$ has closed graph.

By hypothesis, for each $f \in F$ there is $x_{f} \in X$ such that $V_{f}(A)=T\left(x_{f}\right)$. Since $A$ is an atom of $\lambda$, it readily follows that $\left(x_{f} \chi_{A}\right)^{\circ}=\left(f \chi_{A}\right)^{\circ}$. Therefore, $P_{A}\left(F^{*}\right)=\left\{\left(x \chi_{A}\right)^{\circ}: x \in X\right\}$. On the other hand, the fact that $A$ is an atom of $\lambda$ implies that

$$
\|T(x)\|=\sup _{B \in \Sigma}\left\|V_{x_{\chi_{A}}}(B)\right\| \leq\left\|\left(x \chi_{A}\right)^{\bullet}\right\| \leq 2 \sup _{B \in \Sigma}\left\|V_{x \chi_{A}}(B)\right\|=2\|T(x)\|
$$

for every $x \in X$. Thus we can define a linear homeomorphism $\phi: P_{A}\left(F^{*}\right) \longrightarrow T(X)$ by $\phi\left(\left(x \chi_{A}\right)^{\circ}\right):=T(x)$.

Let us turn to the proof of the equivalence between (i)-(iv). The statements (i) implies (ii) implies (iii) are well known general facts, see for instance [22, Section 6.1]. Now assume that (iii) holds. Then $T(X)$ is barrelled and, since $\tilde{T}^{-1}$ has closed graph, an appeal to the closed graph theorem for linear mappings from a barrelled space into a Fréchet space (see for instance [22, Theorem 4.1.10]) establishes that $\tilde{T}^{-1}$ is continuous. Hence $\tilde{T}$ is a linear homeomorphism between the Banach space $X / \operatorname{ker} T$ and $T(X)$. Therefore $T(X)$ is complete and so it is closed in $Y$. This proves (iii) implies (iv). Finally, if $T(X)$ is a closed subspace of $Y$, then $T(X)$ is complete and, since $\phi$ is a linear homeomorphism, $P_{A}\left(F^{*}\right)$ is complete also. The proof is finished.

4.1. Spaces of Dobrakov integrable functions It is not difficult to see that $\left(D(\mu),\|\cdot\|_{I}\right.$ ) belongs to the class $\mathfrak{J}_{\mu}$ (take $V_{f}^{D(\mu)}=I_{f}$ for every $f \in D(\mu)$ ) and that for each atom $A$ of $\hat{\mu}$ we have $I_{f}(A) \in \mu(A)(X)$ for every $f \in D(\mu)$ (bear in mind that a measurable function defined on $A$ must be $\lambda$-essentially constant). As an immediate consequence of Proposition 4.5 we obtain the following corollary.

COROLlARY 4.6. If $A \in \Sigma$ is an atom of $\hat{\mu}$, then $P_{A}\left(D(\mu)^{\circ}\right)$ is linearly homeomorphic to $\mu(A)(X)$ and the following conditions are equivalent: 
(i) $P_{A}\left(D(\mu)^{\circ}\right)$ is a Banach space;

(ii) $P_{A}\left(D(\mu)^{\circ}\right)$ is ultrabornological;

(iii) $P_{A}\left(D(\mu)^{\circ}\right)$ is barrelled;

(iv) $\mu(A)(X)$ is closed in $Y$.

THEOREM 4.7. The following conditions are equivalent:

(i) $D(\mu)^{\bullet}$ is ultrabornological;

(ii) $D(\mu)^{*}$ is barrelled;

(iii) $\mu(A)(X)$ is closed in $Y$ for every atom $A$ of $\hat{\mu}$.

Proof. We already know that (i) implies (ii). To prove (ii) implies (iii) fix an atom $A$ of $\hat{\mu}$ and notice that, since $P_{A}$ is a continuous linear projection, $P_{A}\left(D(\mu)^{\bullet}\right)$ is a complemented subspace of $D(\mu)^{\circ}$, hence $P_{A}\left(D(\mu)^{\circ}\right)$ is barrelled, see [22, Corollary 4.2 .2 (i)]. An appeal to Corollary 4.6 ensures us that $\mu(A)(X)$ is closed in $Y$.

Finally, the statement (iii) implies (i) is established by applying Corollary 4.4 . We already know that $P_{A}\left(D(\mu)^{*}\right)$ is ultrabornological for every atom $A$ of $\hat{\mu}$ (by Corollary 4.6) and so it only remains to check the following fact.

(*) If $\left(A_{n}\right)_{n=1}^{\infty}$ is a countable partition of $\Omega$ in $\Sigma,\left(f_{n}\right)_{n=1}^{\infty}$ is a bounded sequence in $\left(D(\mu),\|\cdot\|_{I}\right)$ such that $\left.f_{n}\right|_{A_{m}} \equiv 0$ whenever $n>m$, and $\left(\alpha_{n}\right)_{n=1}^{\infty} \in \ell^{1}$, then the function $f: \Omega \longrightarrow X$ given by $f:=\sum_{n=1}^{\infty} \alpha_{n} f_{n}$ is Dobrakov integrable with respect to $\mu$ and $\lim _{m}\left\|\sum_{n=1}^{m} \alpha_{n} f_{n}-f\right\|_{I}=0$.

To prove (*) define $g_{m}:=\sum_{n=1}^{m} \alpha_{n} f_{n} \in D(\mu)$ for every $m \in \mathbb{N}$. Observe that $\left(g_{m}\right)_{m=1}^{\infty}$ converges pointwise to $f$ and that for each $E \in \Sigma$ there exists the limit (for the norm topology of $Y) \lim _{m}(D) \int_{E} g_{m} d \mu=\lim _{m} \sum_{n=1}^{m} \alpha_{n} I_{f_{n}}(E)$, because $\left(\alpha_{n}\right)_{n=1}^{\infty} \in \ell^{1}$ and $\sup _{n \in \mathbb{N}}\left\|f_{n}\right\|_{I}<\infty$. An appeal to [6, Theorem 16] ensures that $f \in D(\mu)$ and that $\lim _{m} I_{g_{m}}(E)=I_{f}(E)$ uniformly for $E \in \Sigma$. Hence $\lim _{m}\left\|\sum_{n=1}^{m} \alpha_{n} f_{n}-f\right\|_{I}=0$ and condition (*) holds. Therefore $D(\mu)^{*}$ is ultrabornological and the proof is finished.

It was claimed in [29, Theorem 16] that $D(\mu)^{\bullet}$ is always barrelled. However, this statement is wrong. The simplest example of a non-barrelled $D(\mu)^{\bullet}$ is the range of a bounded operator between Banach spaces provided it is not closed. More precisely, take a non-empty set $\Omega$ and consider the $\sigma$-algebra $\Sigma=\{\emptyset, \Omega\}$. Fix a bounded operator $T$ between the Banach spaces $Z_{1}$ and $Z_{2}$ such that $T\left(Z_{1}\right)$ is not closed in $Z_{2}$ and define $\mu: \Sigma \longrightarrow \mathcal{L}\left(Z_{1}, Z_{2}\right)$ by $\mu(\emptyset):=0$ and $\mu(\Omega):=T$. Then $D(\mu)^{\bullet}$ (that is linearly homeomorphic to $T\left(Z_{2}\right)$ ) is not barrelled.

REMARK. It is elementary that a locally convex space with a dense barrelled linear subspace is barrelled, see for example [22, Proposition 4.2.1 (ii)]. Combining Theo- 
rem 4.7 with Theorem 3.3 (respectively Theorem 3.5) we infer that $S^{*}(\mu)^{\bullet}$ (respectively $M(\mu)^{\circ}$, in contexts in which it is definable) is barrelled provided that $\mu(A)(X)$ is closed in $Y$ for every atom $A$ of $\hat{\mu}$. In the next subsections some extra work leads us to prove that these spaces are even ultrabornological.

4.2. Spaces of $S^{\bullet}$-integrable functions The aim of this subsection is to prove the analogue of Theorem 4.7 for the space of $S^{*}$-integrable functions. The proof requires some previous work. First of all, observe that Lemma 4.8 below guarantees that $\left(S^{*}(\mu),\|\cdot\|_{\nu}\right)$ belongs to the class $\mathfrak{J}_{\mu}$ (take $V_{f}^{S^{*}(\mu)}=\nu_{f}$ for every $f \in S^{*}(\mu)$ ).

LEMMA 4.8. Let $f \in S^{*}(\mu)$ and $A \in \Sigma$. Then $f \chi_{A} \in S^{*}(\mu)$ and $v_{f \chi_{A}}(\Omega)=v_{f}(A)$.

Proof. Fix $\epsilon>0$. Since $\left.f\right|_{A}$ is $S^{*}$-integrable with respect to $\mu_{A}$, there is a countable partition $\Gamma_{0}^{A}$ of $A$ in $\Sigma_{A}$ such that

$$
\left\|S\left(f, \Gamma^{\prime}, T^{\prime}\right)-v_{f}(A)\right\| \leq \epsilon
$$

for every countable partition $\Gamma^{\prime}$ of $A$ in $\Sigma_{A}$ finer than $\Gamma_{0}^{A}$ and every choice $T^{\prime}$ in $\Gamma$, the series $S\left(f, \Gamma^{\prime}, T^{\prime}\right)$ being unconditionally convergent. Define $\Gamma_{0}:=\Gamma_{0}^{A} \cup\{\Omega \backslash A\}$.

If $\Gamma=\left(A_{n}\right)$ is a countable partition of $\Omega$ in $\Sigma$ finer than $\Gamma_{0}$ and $T=\left(t_{n}\right)$ is any choice in $\Gamma$, then $\Gamma^{\prime}=\left\{A_{n}: A_{n} \subset A\right\}$ is a countable partition of $A$ in $\Sigma_{A}$ finer than $\Gamma_{0}^{A}$ and $T^{\prime}=\left\{t_{n}: A_{n} \subset A\right\}$ is a choice in $\Gamma^{\prime}$, hence $S\left(f \chi_{A}, \Gamma, T\right)=S\left(\left.f\right|_{A}, \Gamma^{\prime}, T^{\prime}\right)$ is unconditionally convergent and (4.1) yields

$$
\left\|S\left(f \chi_{A}, \Gamma, T\right)-v_{f}(A)\right\| \leq \epsilon .
$$

As $\epsilon>0$ is arbitrary, $f \chi_{A} \in S^{*}(\mu)$ and $\left(S^{*}\right) \int_{\Omega} f \chi_{A} d \mu=v_{f}(A)$.

COROLlary 4.9. If $A \in \Sigma$ is an atom of $\hat{\mu}$, then $P_{A}\left(S^{*}(\mu)^{\circ}\right)$ is linearly homeomorphic to $\mu(A)(X)$ and the following conditions are equivalent:

(i) $P_{A}\left(S^{*}(\mu)^{\circ}\right)$ is a Banach space;

(ii) $P_{A}\left(S^{*}(\mu)^{\circ}\right)$ is ultrabornological;

(iii) $P_{A}\left(S^{*}(\mu)^{\circ}\right)$ is barrelled;

(iv) $\mu(A)(X)$ is closed in $Y$.

PROOF. The result follows straightforwardly from Proposition 4.5, bearing in mind that $v_{f}(A) \in \mu(A)(X)$ for every $f \in S^{*}(\mu)$ (by Lemma 3.2).

Corollary 4.9 and the comments after Theorem 4.7 make clear that $S^{*}(\mu)^{\bullet}$ is not barrelled in general.

The following criterion of unconditional convergence for double series in Banach spaces will be used in the proofs of Lemmas 4.11 and 4.12 . 
LEMMA 4.10 ([3, Lemma 3.1]). Let $\left(z_{n, k}\right)_{n, k \in \mathbb{N}}$ be a double sequence in a Banach space $Z$ such that

(i) the series $\sum_{k} z_{n, k}$ is unconditionally convergent for every $n \in \mathbb{N}$;

(ii) there exist an unconditionally convergent series $\sum_{n, k} z_{n, k}^{\prime}$ in $Z$ and a sequence $\left(a_{n}\right)_{n=1}^{\infty} \in \ell^{1}$ such that $\left\|\sum_{k \in Q}\left(z_{n, k}-z_{n, k}^{\prime}\right)\right\| \leq\left|a_{n}\right|$ for every finite set $Q \subset \mathbb{N}$ and every $n \in \mathbb{N}$.

Then $\sum_{n, k} z_{n, k}$ is unconditionally convergent in $Z$.

LEMMA 4.11. Let $f: \Omega \longrightarrow X$ be a function. The following conditions are equivalent:

(i) $f$ is $S^{*}$-integrable with respect to $\mu$;

(ii) there is a countable partition $\Gamma_{0}=\left(A_{n}\right)$ of $\Omega$ in $\Sigma$ such that $\left.f\right|_{A_{n}} \in S^{*}\left(\mu_{A_{n}}\right)$ for every $n$, and for every countable partition $\Gamma=\left(B_{n}\right)$ of $\Omega$ in $\Sigma$ finer than $\Gamma_{0}$, the series $\sum_{n}\left(S^{*}\right) \int_{B_{n}} f d \mu_{B_{n}}$ converges unconditionally.

Proof. It only remains to prove (ii) implies (i). We show that $f \in S^{*}(\mu)$ and $\left(S^{*}\right) \int_{\Omega} f d \mu=\sum_{n}\left(S^{*}\right) \int_{A_{n}} f d \mu_{A_{n}}=: y \in Y$. To this end fix $\epsilon>0$. For each $n \in \mathbb{N}$, we apply Lemma 3.1 to obtain a countable partition $\Gamma_{1}^{n}$ of $A_{n}$ in $\Sigma_{A_{n}}$ such that for every countable family $\Gamma^{\prime}=\left(C_{m}\right)$ of pairwise disjoint elements of $\Sigma_{A_{n}}$ finer than $\Gamma_{1}^{n}$ and every choice $T^{\prime}$ in $\Gamma^{\prime}$,

$$
\left\|S\left(f, \Gamma^{\prime}, T^{\prime}\right)-\left(S^{*}\right) \int_{C} f d \mu_{C}\right\| \leq \frac{\epsilon}{2^{n}},
$$

where $C=\bigcup_{m} C_{m}$, the series $S\left(f, \Gamma^{\prime}, T^{\prime}\right)$ being unconditionally convergent.

Observe that $\Gamma_{1}:=\bigcup_{n} \Gamma_{1}^{n}$ is a countable partition of $\Omega$ in $\Sigma$ finer than $\Gamma_{0}$. We claim that if $\Gamma$ is any countable partition of $\Omega$ in $\Sigma$ finer than $\Gamma_{1}$ and $T$ is any choice in $\Gamma$, then the series $S(f, \Gamma, T)$ converges unconditionally and $\|S(f, \Gamma, T)-y\| \leq \epsilon$. Indeed, let $\Gamma=\left(A_{n, k}\right)$ be such a partition, where $\Gamma^{n}:=\left(A_{n, k}\right)_{k}$ is a countable partition of $A_{n}$ in $\Sigma_{A_{n}}$ finer than $\Gamma_{1}^{n}$ for every $n$. Fix any choice $T=\left(t_{n, k}\right)$ in $\Gamma$ and define $T^{n}:=\left(t_{n, k}\right)_{k}$ for every $n$. We first check that $S(f, \Gamma, T)$ is unconditionally convergent with the help of Lemma 4.10. Notice that

(a) the series $S\left(f, \Gamma^{n}, T^{n}\right)$ is unconditionally convergent for every $n$;

(b) $\sum_{n, k}\left(S^{*}\right) \int_{A_{n, k}} f d \mu_{A_{n, k}}$ is unconditionally convergent too, since $\Gamma=\left(A_{n, k}\right)$ is finer than $\Gamma_{0}$;

(c) for each finite set $Q \subset \mathbb{N}$ and each $n \in \mathbb{N}$ inequality (4.2) yields

$$
\left\|\sum_{k \in Q} \mu\left(A_{n, k}\right)\left(f\left(t_{n, k}\right)\right)-\sum_{k \in Q}\left(S^{*}\right) \int_{A_{n, k}} f d \mu_{A_{n, k}}\right\| \leq \frac{\epsilon}{2^{n}} .
$$


An appeal to Lemma 4.10 allows us to conclude that $S(f, \Gamma, T)$ converges unconditionally in $Y$. Finally, (4.2) implies that

$$
\|S(f, \Gamma, T)-y\| \leq \sum_{n}\left\|S\left(f, \Gamma^{n}, T^{n}\right)-\left(S^{*}\right) \int_{A_{n}} f d \mu_{A_{n}}\right\| \leq \sum_{n} \frac{\epsilon}{2^{n}} \leq \epsilon .
$$

As $\epsilon>0$ is arbitrary, $f \in S^{*}(\mu)$ and $\left(S^{*}\right) \int_{\Omega} f d \mu=y$. The proof is complete.

In the particular case of Birkhoff integrable functions, the previous result was proved in [3, Lemma 3.2] using the countable additivity of the indefinite Pettis integral.

LEMMA 4.12. Let $\left(A_{n}\right)_{n=1}^{\infty}$ be a countable partition of $\Omega$ in $\Sigma,\left(f_{n}\right)_{n=1}^{\infty}$ a bounded sequence in $\left(S^{*}(\mu),\|\cdot\|_{v}\right)$ such that $\left.f_{n}\right|_{A_{m}} \equiv 0$ whenever $n>m$, and $\left(\alpha_{n}\right)_{n=1}^{\infty} \in \ell^{l}$. Define $f: \Omega \longrightarrow X$ by $f:=\sum_{n=1}^{\infty} \alpha_{n} f_{n}$. Then

(i) $f \in S^{*}(\mu)$;

(ii) $v_{f}(E)=\sum_{n=1}^{\infty} \alpha_{n} v_{f_{n}}(E)$ for every $E \in \Sigma$;

(iii) $\lim _{N}\left\|\sum_{n=1}^{N} \alpha_{n} f_{n}-f\right\|_{\nu}=0$.

PROOF. (i) For each $n \in \mathbb{N}$ we have $\left.f\right|_{A_{n}}=\left.\sum_{i=1}^{n} \alpha_{i} f_{i}\right|_{A_{n}}$, hence $\left.f\right|_{A_{n}}$ is $S^{*}$ integrable with respect to $\mu_{A_{n}}$. Thus, in view of Lemma 4.11 , in order to prove that $f \in S^{*}(\mu)$ we only have to check that if $\Gamma$ is a countable partition of $\Omega$ in $\Sigma$ finer than $\left(A_{n}\right)_{n=1}^{\infty}$, then the series $\sum_{A \in \Gamma}\left(S^{*}\right) \int_{A} f d \mu$ converges unconditionally in $Y$.

To this end fix such a partition $\Gamma=\left(A_{n, k}\right)$, where $A_{n}=\bigcup_{k} A_{n, k}$ for every $n \in \mathbb{N}$. Notice that

$$
\left(S^{*}\right) \int_{A_{n, k}} f d \mu_{A_{n, k}}=\sum_{i=1}^{n} \alpha_{i} v_{f_{i}}\left(A_{n, k}\right)
$$

for every $n, k \in \mathbb{N}$. Write $K:=\sup _{n \in \mathbb{N}}\left\|f_{n}\right\|_{\nu}$ and fix $\epsilon>0$. There is an $N \in \mathbb{N}$ large enough such that $\left(\sum_{n>N}\left|\alpha_{n}\right|\right) K \leq \epsilon$. Since each $v_{f_{n}}$ is countably additive, there is a finite set $T \subset \mathbb{N} \times \mathbb{N}$ such that $\left|\alpha_{n}\right|\left\|\nu_{f_{n}}\left(\bigcup_{(m, k) \in S} A_{m, k}\right)\right\| \leq \epsilon / N$ for every $1 \leq n \leq N$ whenever $S \subset \mathbb{N} \times \mathbb{N}$ is finite and $S \cap T=\emptyset$. Combining (4.3) with the inequalities above we infer

$$
\begin{aligned}
\left\|\sum_{(m, k) \in S}\left(S^{*}\right) \int_{A_{m, k}} f d \mu_{A_{m, k}}\right\| & \left\|\sum_{(m, k) \in S} \sum_{i=1}^{m} \alpha_{i} v_{f_{i}}\left(A_{m, k}\right)\right\|=\left\|\sum_{n=1}^{\infty} \sum_{\substack{(m, k) \in S \\
n \leq m}} \alpha_{n} v_{f_{n}}\left(A_{m, k}\right)\right\| \\
\leq & \sum_{n=1}^{N}\left|\alpha_{n}\right|\left\|v_{f_{n}}\left(\bigcup_{\substack{(m, k) \in S \\
n \leq m}} A_{m, k}\right)\right\|+\sum_{n>N}\left|\alpha_{n}\right|\left\|v_{f_{n}}\left(\bigcup_{\substack{(m, k) \in S \\
n \leq m}}^{\bigcup} A_{m, k}\right)\right\|
\end{aligned}
$$




$$
\leq \sum_{n=1}^{N} \frac{\epsilon}{N}+\left(\sum_{n>N}\left|\alpha_{n}\right|\right) K \leq 2 \epsilon
$$

whenever $S \subset \mathbb{N} \times \mathbb{N}$ is finite and $S \cap T=\emptyset$. As $\epsilon>0$ is arbitrary, the series $\sum_{n, k}\left(S^{*}\right) \int_{A_{n, k}} f d \mu_{A_{n, k}}$ converges unconditionally. This completes the proof of (i).

In order to see (ii) we begin with the claim: The series $\sum_{n, k} \alpha_{n} \nu_{f_{n}}\left(A_{k}\right)$ is unconditionally convergent.

Indeed, this follows immediately from Lemma 4.10 because

(a) the series $\sum_{k} \alpha_{n} v_{f_{n}}\left(A_{k}\right)$ converges unconditionally for every $n \in \mathbb{N}$;

(b) for every finite set $Q \subset \mathbb{N}$ and every $n \in \mathbb{N}$ we have

$$
\left\|\sum_{k \in Q} \alpha_{n} v_{f_{n}}\left(A_{k}\right)\right\|=\| \alpha_{n} v_{f_{n}}\left(\bigcup_{k \in Q} A_{k}\right)|\leq| \alpha_{n} \mid K .
$$

Bearing in mind (4.3), we conclude that

$$
\begin{aligned}
\sum_{n=1}^{\infty} \alpha_{n} v_{f_{n}}(\Omega) & =\sum_{n=1}^{\infty}\left(\sum_{k=1}^{\infty} \alpha_{n} v_{f_{n}}\left(A_{k}\right)\right)=\sum_{k=1}^{\infty}\left(\sum_{n=1}^{\infty} \alpha_{n} v_{f_{n}}\left(A_{k}\right)\right) \\
& =\sum_{k=1}^{\infty}\left(\sum_{n=1}^{k} \alpha_{n} v_{f_{n}}\left(A_{k}\right)\right)=\sum_{k=1}^{\infty} v_{f}\left(A_{k}\right)=v_{f}(\Omega) .
\end{aligned}
$$

Given $E \in \Sigma$, the same argument applied to $\left(\left.f_{n}\right|_{E}\right)_{n=1}^{\infty}$ and $\left(A_{n} \cap E\right)_{n=1}^{\infty}$ yields $v_{f}(E)=\sum_{n=1}^{\infty} \alpha_{n} v_{f_{n}}(E)$, as required.

Finally, to deduce (iii) fix $\epsilon>0$ and take $N_{0} \in \mathbb{N}$ large enough such that $\sum_{n>N_{0}}\left|\alpha_{n}\right| \leq \epsilon$. For each $N \geq N_{0}$ the function $h_{N} \in S^{*}(\mu)$ defined by

satisfies

$$
h_{N}:=f-\sum_{n=1}^{N} \alpha_{n} f_{n}=\sum_{n>N} \alpha_{n} f_{n}
$$

$$
\left\|h_{N}\right\|_{\nu} \leq 2 \sup _{E \in \Sigma}\left\|v_{h_{N}}(E)\right\|=2 \sup _{E \in \Sigma}\left\|\sum_{n>N} \alpha_{n} v_{f_{n}}(E)\right\| \leq 2 K\left(\sum_{n>N}\left|\alpha_{n}\right|\right) \leq 2 K \epsilon,
$$

where the equality follows from (ii) and the linearity of the $S^{*}$-integral. As $\epsilon>0$ is arbitrary, (iii) holds and the proof is finished.

We can now prove the chief result of this subsection.

THEOREM 4.13. The following conditions are equivalent:

(i) $S^{*}(\mu)^{\bullet}$ is ultrabornological; 
(ii) $S^{*}(\mu)^{\bullet}$ is barrelled;

(iii) $\mu(A)(X)$ is closed in $Y$ for every atom $A$ of $\hat{\mu}$.

PROOF. The proof of (ii) implies (iii) follows the same steps as for $D(\mu)^{\circ}$ in Theorem 4.7, but now using Corollary 4.9 instead of Corollary 4.6. On the other hand, the statement (iii) implies (i) is a straightforward consequence of Corollaries 4.4 and 4.9 and Lemma 4.12 .

In the particular case of integration with respect to a non-negative finite measure (see case (C1) in Subsection 2.1) condition (iii) in Theorem 4.13 is automatically fulfilled. Consequently, we obtain the following result for spaces of Birkhoff integrable functions.

COROLLARY 4.14. Let $(T, \mathcal{A}, \theta)$ be a complete probability space and $Z$ a Banach space. Then the space of all (equivalence classes of) Birkhoff integrable functions defined on $T$ with values in $Z$, endowed with the Pettis norm, is ultrabornological.

4.3. Spaces of McShane integrable functions In this subsection we analyze when the space of McShane integrable functions is ultrabornological. Theorem 4.20 below shows that the same conclusions which we have already obtained for $D(\mu)^{\circ}$ and $S^{*}(\mu)^{\circ}$ remain true for $M(\mu)^{\circ}$. In the sequel $\tau$ is a topology on $\Omega$ with $\tau \subset \Sigma$ and we suppose that $\hat{\mu}$ fulfills properties $(\alpha),(\beta)$ and $(\gamma)$ mentioned in Subsection 2.2.

From the next two lemmas it will become clear that $\left(M(\mu),\|\cdot\|_{\zeta}\right)$ is a seminormed space in the class $\mathfrak{J}_{\mu}$ (with $V_{f}^{M(\mu)}=\zeta_{f}$ for every $f \in M(\mu)$ ) that satisfies the requirements in Proposition 4.5.

LEMMA 4.15 ([26], Proposition 3.4). Let $f \in M(\mu)$ and $A \in \Sigma$. Then $f \chi_{A} \in$ $M(\mu)$ and $\zeta_{f \chi_{A}}(\Omega)=\zeta_{f}(A)$.

LEMMA 4.16. Suppose that $\Omega$ is an atom of $\hat{\mu}$. If $f \in M(\mu)$, then there is $E \in \Sigma$ such that $\hat{\mu}(\Omega \backslash E)=0$ and $\zeta_{f}(\Omega)=\mu(\Omega)(f(\omega))$ for every $\omega \in E$.

Proof. The family $\mathcal{G}$, made up of all $G \in \tau$ with $\lambda(G)=0$, is non-empty and upwards directed, hence property $(\beta)$ may be applied to deduce that $\lambda(\bigcup \mathcal{G})=0$. Define $E:=\Omega \backslash \bigcup \mathcal{G}$. Since $\left.f\right|_{E}$ is McShane integrable with respect to $\mu_{E}$, with integral $\zeta_{f}(E)=\zeta_{f}(\Omega)$, for each $n \in \mathbb{N}$ there exist a gauge $\delta_{n}$ on $\left(E, \tau_{E}\right)$ and $\eta_{n}>0$ such that

$$
\left\|\sum_{i=1}^{p} \mu\left(E_{i}\right)\left(f\left(s_{i}\right)\right)-\zeta_{f}(\Omega)\right\| \leq \frac{1}{n}
$$


for every partial McShane partition $\left\{\left(E_{i}, s_{i}\right): 1 \leq i \leq p\right\}$ of $E$ subordinate to $\delta_{n}$ such that $\hat{\mu}\left(E \backslash \bigcup_{i=1}^{p} E_{i}\right) \leq \eta_{n}$.

Fix $\omega \in E$ and $n \in \mathbb{N}$. Then $\delta_{n}(\omega)=G_{n} \cap E=G_{n} \backslash \bigcup \mathcal{G}$ for some $G_{n} \in \tau$. Since $\delta_{n}(\omega) \neq \emptyset$, we have $\lambda\left(G_{n}\right)>0$ and, since $\Omega$ is an atom of $\lambda$, it follows that $\lambda\left(E \backslash \delta_{n}(\omega)\right)=0$. In particular, $\mu\left(\delta_{n}(\omega)\right)=\mu(\Omega)$. Therefore (4.4) applied to $\left\{\left(\delta_{n}(\omega), \omega\right)\right\}$ yields $\left\|\mu(\Omega)(f(\omega))-\zeta_{f}(\Omega)\right\| \leq 1 / n$. As $\omega \in E$ and $n \in \mathbb{N}$ are arbitrary, we deduce that $\zeta_{f}(\Omega)=\mu(\Omega)(f(\omega))$ for every $\omega \in E$ and the proof is over.

COROLlARY 4.17. If $A \in \Sigma$ is an atom of $\hat{\mu}$, then $P_{A}\left(M(\mu)^{\circ}\right)$ is linearly homeomorphic to $\mu(A)(X)$ and the following conditions are equivalent:

(i) $P_{A}\left(M(\mu)^{*}\right)$ is a Banach space;

(ii) $P_{A}\left(M(\mu)^{\circ}\right)$ is ultrabornological;

(iii) $P_{A}\left(M(\mu)^{\circ}\right)$ is barrelled;

(iv) $\mu(A)(X)$ is closed in $Y$.

In order to apply Corollary 4.4 to $\left(M(\mu),\|\cdot\|_{\zeta}\right)$, we also need Proposition 4.18 below. The proof of this Beppo-Levi type result is inspired by some of the ideas used in [27, Theorem 8] and [28, Theorem 6] for the McShane integral of vector-valued functions defined on $\mathbb{R}$.

PROPOSITION 4.18. Let $\left(f_{n}\right)$ be a sequence in $M(\mu)$ such that $\sum_{n=1}^{\infty} f_{n}$ converges pointwise for the norm topology of $X$, and $\sum_{n=1}^{\infty}\left\|f_{n}\right\|_{\zeta}<\infty$. Then

(i) $f:=\sum_{n=1}^{\infty} f_{n}$ is McShane integrable with respect to $\mu$;

(ii) $\zeta_{f}(E)=\sum_{n=1}^{\infty} \zeta_{f}(E)$ for every $E \in \Sigma$;

(iii) $\lim _{N}\left\|\sum_{n=1}^{N} f_{n}-f\right\|_{\zeta}=0$.

PROOF. Define $g_{n}:=\sum_{k=1}^{n} f_{k} \in M(\mu)$ for every $n \in \mathbb{N}$ and fix $\epsilon>0$. For each $n \in \mathbb{N}$ the Henstock-Saks Lemma [26, Lemma 3.3] can be used to obtain a gauge $\delta_{n}$ on $(\Omega, \tau)$ such that

$$
\left\|g_{n}(\mathcal{P})-\zeta_{g_{n}}\left(W_{\mathcal{P}}\right)\right\| \leq \epsilon / 2^{n}
$$

for every partial McShane partition $\mathcal{P}$ of $\Omega$ subordinate to $\delta_{n}$. Fix $N \in \mathbb{N}$ large enough such that

$$
\sum_{n>N}\left\|f_{n}\right\|_{\zeta} \leq \epsilon
$$

Since for each $n \in \mathbb{N}$ we have $\lim _{\hat{\mu}(E) \rightarrow 0} \zeta_{f_{n}}(E)=0$ (see [26, Lemma 3.2]), there is $\eta>0$ such that

$$
\left\|\zeta_{f_{n}}(E)\right\| \leq \epsilon / 2^{n} \text { for every } 1 \leq n \leq N
$$


whenever $\hat{\mu}(E) \leq \eta$. On the other hand, given $\omega \in \Omega$, fix $N<n(\omega) \in \mathbb{N}$ such that $\left\|g_{n}(\omega)-f(\omega)\right\| \leq \epsilon$ for every $n \geq n(\omega)$.

Let us define a gauge $\delta$ on $(\Omega, \tau)$ by $\delta(\omega):=\delta_{n(\omega)}(\omega)$ for every $\omega \in \Omega$. We claim that

$$
\left\|f(\mathcal{P})-\sum_{n=1}^{\infty} \zeta_{f_{n}}(\Omega)\right\| \leq(\hat{\mu}(\Omega)+3) \epsilon \quad \text { for every } \mathcal{P} \in \Pi_{\delta, \eta} .
$$

Indeed, take any $\mathcal{P}=\left\{\left(E_{i}, s_{i}\right): 1 \leq i \leq p\right\} \in \Pi_{\delta, \eta}$. For each $n \in \mathbb{N}$ the collection $\left\{\left(E_{i}, s_{i}\right): n\left(s_{i}\right)=n\right\}$ is a (maybe empty) partial McShane partition of $\Omega$ subordinate to $\delta_{n}$, hence (4.5) yields

$$
\begin{aligned}
& \left\|\sum_{i=1}^{p} \mu\left(E_{i}\right) g_{n\left(s_{i}\right)}\left(s_{i}\right)-\sum_{i=1}^{p} \zeta_{g_{n\left(s_{i}\right)}}\left(E_{i}\right)\right\| \\
& \quad \leq \sum_{n=1}^{\infty}\left\|\sum_{n\left(s_{i}\right)=n} \mu\left(E_{i}\right) g_{n}\left(s_{i}\right)-\zeta_{g_{n}}\left(\bigcup_{n\left(s_{i}\right)=n} E_{i}\right)\right\| \\
& \quad \leq \sum_{n=1}^{\infty} \frac{\epsilon}{2^{n}}=\epsilon .
\end{aligned}
$$

The choice of $n\left(s_{i}\right)$ implies $\left\|g_{n\left(s_{i}\right)}\left(s_{i}\right)-f\left(s_{i}\right)\right\| \leq \epsilon$ for every $1 \leq i \leq p$, and therefore

$$
\begin{aligned}
\left\|\sum_{i=1}^{p} \mu\left(E_{i}\right) f\left(s_{i}\right)-\sum_{i=1}^{p} \zeta_{g_{n\left(s_{i}\right)}}\left(E_{i}\right)\right\| & \leq\left\|\sum_{i=1}^{p} \mu\left(E_{i}\right)\left(f\left(s_{i}\right)-g_{n\left(s_{i}\right)}\left(s_{i}\right)\right)\right\|+\epsilon \\
& \leq(\hat{\mu}(\Omega)+1) \epsilon .
\end{aligned}
$$

On the other hand, (4.6) and (4.7) yield

$$
\begin{aligned}
\left\|\sum_{i=1}^{p} \zeta_{g_{n\left(s_{i}\right)}}\left(E_{i}\right)-\sum_{n=1}^{\infty} \zeta_{f_{n}}(\Omega)\right\| & =\left\|\sum_{i=1}^{p} \sum_{k=1}^{n\left(s_{i}\right)} \zeta_{f_{k}}\left(E_{i}\right)-\sum_{n=1}^{\infty} \zeta_{f_{n}}(\Omega)\right\| \\
& =\left\|\sum_{n=1}^{\infty} \zeta_{f_{n}}\left(\bigcup_{n\left(s_{i}\right) \geq n} E_{i}\right)-\sum_{n=1}^{\infty} \zeta_{f_{n}}(\Omega)\right\| \\
& \leq \sum_{n=1}^{N}\left\|\zeta_{f_{n}}\left(\Omega \backslash \bigcup_{i=1}^{p} E_{i}\right)\right\|+\epsilon \\
& \leq \sum_{n=1}^{N} \frac{\epsilon}{2^{n}}+\epsilon \leq 2 \epsilon,
\end{aligned}
$$

keeping in mind that $n\left(s_{i}\right)>N$ for every $1 \leq i \leq p$ and that $\hat{\mu}\left(\Omega \backslash \bigcup_{i=1}^{p} E_{i}\right) \leq \eta$. Inequality (4.8) now follows from the previous one and (4.9). As $\epsilon>0$ is arbitrary, 
$f \in M(\mu)$ and $\zeta_{f}(\Omega)=\sum_{n=1}^{\infty} \zeta_{f_{n}}(\Omega)$. This completes the proof of (i). Statement (ii) can be obtained by applying the previous arguments to each $\left.f\right|_{E}$ with $E \in \Sigma$.

Finally, we prove (iii). Given $N \in \mathbb{N}$, the function

$$
f-\sum_{n=1}^{N} f_{n}=\sum_{n>N} f_{n}=: h_{N} \in M(\mu)
$$

satifies $\zeta_{h_{N}}(E)=\sum_{n>N} \zeta_{f_{n}}(E)$ for every $E \in \Sigma$, by (ii). Hence

$$
\begin{aligned}
\frac{1}{2}\left(\limsup \left\|h_{N}\right\|_{\zeta}\right) & \leq \limsup \sup _{E \in \Sigma}\left\|\zeta_{h_{N}}(E)\right\| \\
& =\lim \sup _{N} \sup _{E \in \Sigma}\left\|\sum_{n>N} \zeta_{f_{n}}(E)\right\| \leq \lim _{N} \sum_{n>N}\left\|f_{n}\right\|_{\zeta}=0 .
\end{aligned}
$$

Therefore, (iii) holds true and the proof is complete.

REMARK. The analogue of Proposition 4.18 for the Birkhoff integral does not hold in general. Indeed, let us consider the sequence of Birkhoff integrable functions $f_{n}:[0,1] \longrightarrow c_{0}([0,1])$ constructed in $[25$, Theorem 2.5$]$ that converges pointwise to a function $f:[0,1] \longrightarrow c_{0}([0,1])$ which is not Birkhoff integrable. A glance at the proofs of Theorems 2.3 and 2.5 in [25] reveals that each $f_{n}$ is scalarly null. Define $g_{1}:=f_{1}$ and $g_{n}:=f_{n}-f_{n-1}$ for every $n \geq 2$. Then $g_{n}$ is Birkhoff integrable and scalarly null for every $n \in \mathbb{N}$. However, $\sum_{n=1}^{\infty} g_{n}=\lim _{n} f_{n}=f$ is not Birkhoff integrable.

COROLLARY 4.19. Let $\left(A_{n}\right)_{n=1}^{\infty}$ be a countable partition of $\Omega$ in $\Sigma,\left(f_{n}\right)_{n=1}^{\infty}$ a bounded sequence in $\left(M(\mu),\|\cdot\|_{\zeta}\right)$ such that $\left.f_{n}\right|_{A_{m}} \equiv 0$ whenever $n>m$, and $\left(\alpha_{n}\right)_{n=1}^{\infty} \in \ell^{1}$. Define $f: \Omega \longrightarrow X$ by $f:=\sum_{n=1}^{\infty} \alpha_{n} f_{n}$. Then

(i) $f \in M(\mu)$;

(ii) $\lim _{N}\left\|\sum_{n=1}^{N} \alpha_{n} f_{n}-f\right\|_{\zeta}=0$.

We are thus led to the previously announced result.

THEOREM 4.20. The following conditions are equivalent:

(i) $M(\mu)^{\circ}$ is ultrabornological;

(ii) $M(\mu)^{\circ}$ is barrelled;

(iii) $\mu(A)(X)$ is closed in $Y$ for every atom $A$ of $\hat{\mu}$.

ProOF. Imitate the proof of Theorem 4.13 using Corollaries 4.19 and 4.17 instead of Lemma 4.12 and Corollary 4.9 , respectively. 
COROLlARY 4.21. Let $(T, \mathcal{T}, \mathcal{A}, \theta)$ be a quasi-Radon probability space and $Z$ a Banach space. Then the space of all (equivalence classes of) McShane integrable functions defined on $T$ with values in $Z$, endowed with the Pettis norm, is ultrabornological.

Finally, notice that the example after Theorem 4.7 (with $\tau:=\{\emptyset, \Omega\}$ ) shows that $M(\mu)^{*}$ is not barrelled in general.

\section{References}

[1] R. G. Bartle, 'A general bilinear vector integral', Studia Math. 15 (1956), 337-352.

[2] G. Birkhoff, 'Integration of functions with values in a Banach space', Trans. Amer. Math. Soc. 38 (1935), 357-378.

[3] B. Cascales and J. Rodríguez, 'The Birkhoff integral and the property of Bourgain', Math. Ann. 331 (2005), 259-279.

[4] S. Díaz, A. Fernández, M. Florencio and P. J. Paúl, 'A wide class of ultrabornological spaces of measurable functions', J. Math. Anal. Appl. 190 (1995), 697-713.

[5] J. Diestel and J. J. Uhl, Jr., Vector measures, Math. Surveys 15 (American Mathematical Society, Providence, RI, 1977).

[6] I. Dobrakov, 'On integration in Banach spaces. I', Czechoslovak Math. J. 20 (95) (1970), 511-536.

[7] —_ ' 'On representation of linear operators on $C_{0}(T, \mathbf{X})$ ', Czechoslovak Math. J. 21 (96) (1971), 13-30.

[8] __ 'On integration in Banach spaces. VII', Czechoslovak Math. J. 38 (113) (1988), 434-449.

[9] D. H. Fremlin, 'The McShane and Birkhoff integrals of vector-valued functions', Research report 92-10, version of 13.10.04 (Mathematics Department, University of Essex, Colchester, UK), available at http://www.essex.ac.uk/maths/staff/fremlin/preprints.htm.

[10] — 'Problem ET', version of 27.10.04 (Mathematics Department, University of Essex, Colchester, UK), available at http://www.essex.ac.uk/maths/staff/fremlin/problems.htm.

[11] _ _ 'The generalized McShane integral', Illinois J. Math. 39 (1995), 39-67.

[12] _- Measure theory. Vol. 4 Topological measure spaces (Torres Fremlin, Colchester, 2003).

[13] D. H. Fremlin and M. Talagrand, 'A decomposition theorem for additive set-functions, with applications to Pettis integrals and ergodic means', Math. Z. 168 (1979), 117-142.

[14] F. J. Freniche and J. C. García-Vázquez, 'The Bartle bilinear integration and Carleman operators', J. Math. Anal. Appl. 240 (1999), 324-339.

[15] J. L. Gámez, Denjoy integrals of functions with values in Banach spaces (Ph.D. Thesis, Universidad Complutense de Madrid, Spain, 1997), (Spanish).

[16] A. Gilioli, 'Natural ultrabornological, noncomplete, normed function spaces', Arch. Math. (Basel) 61 (1993), 465-477, Edited by Klaus Floret and Chaim S. Hönig.

[17] L. Janicka and N. J. Kalton, 'Vector measures of infinite variation', Bull. Acad. Polon. Sci. Sér. Sci. Math. Astronom. Phys. 25 (1977), 239-241.

[18] H. Jarchow, Locally convex spaces (B. G. Teubner, Stuttgart, 1981).

[19] K. Musial, 'Topics in the theory of Pettis integration', Rend. Istit. Mat. Univ. Trieste 23 (1991), $177-262$.

[20] T. V. Panchapagesan, 'On the distinguishing features of the Dobrakov integral', Divulg. Mat. 3 (1995), 79-114. 
[21] P. J. Paúl, 'The space of Denjoy-Dunford integrable functions is ultrabornological', Bull. Belg. Math. Soc. Simon Stevin 8 (2001), 75-82.

[22] P. Pérez Carreras and J. Bonet, Barrelled locally convex spaces, North-Holland Math. Studies 131 (North-Holland, Amsterdam, 1987).

[23] B. J. Pettis, 'On integration in vector spaces', Trans. Amer. Math. Soc. 44 (1938), 277-304.

[24] L. Di Piazza and D. Preiss, 'When do McShane and Pettis integrals coincide?', Illinois J. Math. 47 (2003), $1177-1187$.

[25] J. Rodriguez, 'On the existence of Pettis integrable functions which are not Birkhoff integrable', Proc. Amer. Math. Soc. 133 (2005), 1157-1163.

[26] J. Rodríguez, 'On integration of vector functions with respect to vector measures', Czechoslovak Math. J. 56 (2006), 805-825.

[27] C. Swartz, 'Beppo Levi's theorem for the vector-valued McShane integral and applications', Bull. Belg. Math. Soc. Simon Stevin 4 (1997), 589-599.

[28] — , 'Uniform integrability and mean convergence for the vector-valued McShane integral', Real Anal. Exchange 23 (1997/98), 303-311.

[29] —_ 'Barrelledness of the space of Dobrakov integrable functions', Math. Slovaca 51 (2001), $521-528$

[30] G. E. F. Thomas, 'Totally summable functions with values in locally convex spaces', in: Measure theory (Proc. Conf., Oberwolfach, 1975), Lecture Notes in Math. 541 (Springer, Berlin, 1976) pp. 117-131.

Departamento de Matemáticas

Universidad de Murcia

30.100 Espinardo

Murcia

Spain

e-mail: joserr@um.es 